\title{
Non-perturbative to perturbative QCD via the FFBRST
}

\author{
Haresh Raval ${ }^{1,2, a}$, Bhabani Prasad Mandal ${ }^{2, b}$ \\ ${ }^{1}$ Department of Physics, Indian Institute of Technology, Bombay, Mumbai 400076, India \\ 2 Department of Physics, Institute of Science, Banaras Hindu University, Varanasi 221005, India
}

Received: 24 March 2018 / Accepted: 19 May 2018 / Published online: 25 May 2018

(C) The Author(s) 2018

\begin{abstract}
Recently a new type of quadratic gauge was introduced in QCD in which the degrees of freedom are suggestive of a phase of abelian dominance. In its simplest form it is also free of Gribov ambiguity. However this gauge is not suitable for usual perturbation theory. The finite field dependent BRST (FFBRST) transformation is a method established to interrelate generating functionals for different effective versions of gauge fixed field theories. In this paper we propose a FFBRST transformation suitable for transforming the theory in the new quadratic gauge into the standard Lorenz gauge Faddeev-Popov version of the effective lagrangian. The task is made interesting by the fact that the effective lagrangian is invariant under two different BRST transformations which leads to suitable extension of the previous procedures to accomplish the required result. We are thus able to identify a field redefinition to go from a non-perturbative phase of QCD to perturbative QCD.
\end{abstract}

\section{Introduction}

Extensions of the usual Lorenz gauge by including the next order terms quadratic in gauge fields have been studied in several contexts $[1-6]$. In $[7,8]$ it was shown that a purely quadratic gauge condition without the linear terms leads to a suggestive effective lagrangian giving masses to off-diagonal gluons. The consequences of such a condition to lifting the Gribov ambiguity were further studied in [7-9]. The new type of quadratic gauge condition is at first introduced as follows,

$H^{a}\left[A^{\mu}(x)\right]=A_{\mu}^{a}(x) A^{\mu a}(x)=f^{a}(x) ;$ for each $a$

where $f^{a}(x)$ is an arbitrary function of $x$. Several proposals to establish abelian dominance in the infrared (IR) use what is called Abelian Projection [10]. Such and other algebraic

\footnotetext{
a e-mail: haresh@phy.iitb.ac.in

b e-mail: bhabani.mandal@gmail.com
}

gauges are usually non-covariant. But as introduced in $[7,8]$ the above gauge is in fact covariant. The gauge prima facie is an ambiguity free gauge as it is algebraic in nature. Thus, the quadratic gauge shares the same property of being free of Gribov copies as the axial gauges $n_{\mu} A^{\mu a}=f^{a}(x)$ and the flow gauge $\alpha A_{0}^{a}=\nabla \cdot \vec{A}^{a}[11]$ despite being non linear. The Faddeev-Popov determinant in this gauge is given by

$\operatorname{det}\left(\frac{\delta\left(A_{\mu}^{a \epsilon} A^{\mu a \epsilon}\right)}{\delta \epsilon^{b}}\right)=\operatorname{det}\left(2 A_{\mu}^{a}\left(\partial^{\mu} \delta^{a b}-g f^{a c b} A^{\mu c}\right)\right)$,

Therefore, the resulting effective Lagrangian density contains gauge fixing and ghost terms as follows,

$\mathcal{L}_{\mathrm{GF}}+\mathcal{L}_{\text {ghost }}=-\frac{1}{2 \zeta} \sum_{a}\left(A_{\mu}^{a} A^{\mu a}\right)^{2}-2 \sum_{a} \overline{c^{a}} A^{\mu a}\left(D_{\mu} c\right)^{a}$,

where $\zeta$ is an arbitrary gauge fixing parameter and $\left(D_{\mu} c\right)^{a}=$ $\partial_{\mu} c^{a}-g f^{a b c} A_{\mu}^{b} c^{c}$. Now onwards, we shall drop the summation symbol, but the summation over an index $a$ will be understood when it appears repeatedly, including when repeated thrice as in the ghost terms above. In particular,

$-\overline{c^{a}} A^{\mu a}\left(D_{\mu} c\right)^{a}=-\overline{c^{a}} A^{\mu a} \partial_{\mu} c^{a}+g f^{a b c} \overline{c^{a}} c^{c} A^{\mu a} A_{\mu}^{b}$

where the summation over indices $a, b$ and $c$ each runs independently over 1 to $N^{2}-1$. We should note that ghost Lagrangian does not have kinetic terms and hence the ghosts do not propagate in this theory and make no loop contributions. They act like auxiliary fields, but playing an important role in the IR. With this understanding, we write the full effective Lagrangian density in this quadratic gauge as

$$
\begin{aligned}
\mathcal{L}_{Q}= & -\frac{1}{4} F_{\mu \nu}^{a} F^{\mu \nu a}-\frac{1}{2 \zeta}\left(A_{\mu}^{a} A^{\mu a}\right)^{2}-2 \overline{c^{a}} A^{\mu a}\left(D_{\mu} c\right)^{a} \\
= & -\frac{1}{4} F_{\mu \nu}^{a} F^{\mu \nu a}+\frac{\zeta}{2} F^{a 2}+F^{a} A_{\mu}^{a} A^{\mu a} \\
& -2 \overline{c^{a}} A^{\mu a}\left(D_{\mu} c\right)^{a},
\end{aligned}
$$


where the field strength $F_{\mu \nu}^{a}=\partial_{\mu} A_{\nu}^{a}(x)-\partial_{\nu} A_{\mu}^{a}(x)-$ $g f^{a b c} A_{\mu}^{b}(x) A_{\nu}^{c}(x)$ and in the second version the $F^{a}$ are a set of auxiliary fields called Nakanishi-Lautrup fields [12]. As shown in [9], the Lagrangian is BRST invariant [13,14] which is essential for the ghost independence of the green functions and unitarity of the $S$-matrix. These issues were studied in Refs. [7,8].

The form of the second term of the expression (4) appearing in the ghost lagrangian contains ghost bilinears multiplying terms quadratic in gauge fields. Hence if the nonpropagating ghosts are assumed to be frozen they amount to a non-zero mass matrix for the gluons. To strengthen this connection it is necessary to assume that the vacuum corresponds to ghost condensation. This was achieved through introducing a Lorenz gauge fixing term for one of the diagonal gluons, in addition to the purely quadratic terms of Eq. (1). This gauge fixing gives the propagator to the corresponding ghost field. Using this ghost propagator, one can give nontrivial vacuum values to bilinears $\overline{c^{a}} c^{c}$ within the framework Coleman-Weinberg mechanism as described in $[7,8]$. We shall revisit the point in the next section also.

The resulting mass matrix for the gluons has $N(N-1)$ non-zero eigenvalues only and thus has nullity $N-1$. Thus, the $N(N-1)$ off-diagonal gluons acquire masses and the rest $N-1$ diagonal gluons remain massless. The massive off-diagonal gluons are presumed to provide evidence of Abelian dominance, which is a signature of quark confinement. This and other phenomena that emerge in this gauge, such as the avoidance of Gribov ambiguity were studied explicitly in [7-9]. Quark confinement and Gribov ambiguity are important non-perturbative issues. And this gauge therefore proves to be important in studying non-perturbative regime of QCD.

The finite field dependent BRST (FFBRST) transformation was introduced for first time in Ref. [6] by integrating infinitesimal usual BRST transformations. Such FFBRST transformations have exactly the same form as the infinitesimal ones, with the difference that the infinitesimal global anti-commuting parameter is replaced by an anti-commuting but finite parameter dependent on space time fields, but with no explicit dependence on space time coordinates. The meaning of "finite anti-commuting parameter" is that if we calculate the Green's functions for such parameters between vacuum and a state with gauge and ghost fields we get finite values as opposed to infinitesimal values. Being finite in nature FFBRST transformation does not leave the path integral measure invariant even though other properties of usual infinitesimal BRST transformation are intact. Thus the generating functional to a BRST invariant theory is not invariant under FFBRST. Jacobian of such finite transformation provides a non-trivial factor which depends on FFBRST parameter.

Due to this non-trivial Jacobian FFBRST transformations are simultaneously field redefinitions as well as BRST transformations on the fields being redefined. They are thus capable of connecting generating functionals of two different BRST invariant theories and have been used to study different gauge field theoretic models with various effective actions [15-28]. In this paper we construct an appropriate FFBRST transformation to establish the connection at the level of generating functionals between the recently introduced quadratic gauge with substantial implications in the non-perturbative QCD [7-9] and the familiar Lorenz gauge which is suitable to describe the perturbative QCD. This is novel connection since previous connections were either between two gauges suitable for only perturbative sector e.g., connection between Lorenz and axial gauges or they had no such unique field theoretic meaning attached to them. We should here mention that the same FFBRST however does not explicitly connect the vacuum in the quadratic gauge with which non-Perturbative phase of QCD is associated to the vacuum in Lorenz gauge to which perturbative phase of QCD corresponds. To understand this, we first discuss the vacua of both the theories.

As discussed above, the vacuum in the quadratic gauge is provided by the $S U(N)$ symmetric ghost condensation of bilinears $[7,8]$ which is non perturbative in nature since the non perturbative confining phase corresponds to this vacuum $[7,8]$ and it arises at Lagrangian level only as is clear from Eq. (4). Such a vacuum does not exist in the theory of Lorenz gauge. The vacuum in the Lorenz gauge is provided by the mix condensate of gluon and ghost, $\left\langle F^{\mu \nu} F_{\mu \nu}+\bar{c} c\right\rangle$ arising from the following sorts of loops (Fig. 1) [29].

Nature of this vacuum is perturbative as it arises out of loops and, over and above it formal perturbation theory of QCD is built. Contribution of these loops is however trivial at the tree level $\mathcal{O}\left(g^{0}\right)$ since it vanishes in the dimensional
Fig. 1 Loops which can contribute to the vacuum in the Lorenz gauge at lower orders

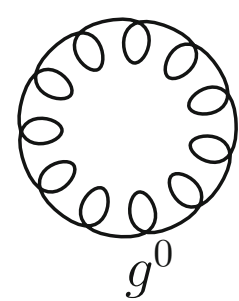

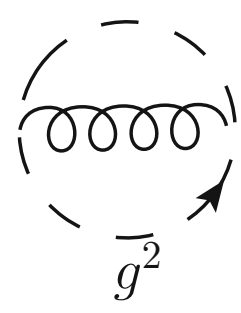


regularization scheme as the gluon propagator is $\frac{1}{p^{2}}$. Therefore, the vacuum in the Lorenz gauge, $\left\langle F^{\mu v} F_{\mu \nu}+\bar{c} c\right\rangle$ is trivial i.e., $\left\langle F^{\mu v} F_{\mu \nu}+\bar{c} c\right\rangle=0$ at tree level. Such vacuum composed of mix condensate does not exist in the theory of the quadratic gauge as the two point gluon and ghost functions are formally absent in the theory. Thus, we can see that confining phase of theory in the quadratic gauge and free gluon phase in the Lorenz gauge belong to vacua of entirely different nature. It is clear that the FFBRST technique based on the $\Theta^{\prime}$ parameter constructed in Eq. (18) explicitly does not connect two vacua discussed above,

$$
\begin{aligned}
& S U(N) \text { symmetric }\langle\bar{c} c\rangle \text { (in the quadratic gauge) } \\
& \stackrel{F F B R S T}{\nrightarrow}\left\langle F^{\mu \nu} F_{\mu \nu}+\bar{c} c\right\rangle \text { (in the Lorenz gauge). }
\end{aligned}
$$

This conclusion is not at all surprising as the FFBRST is designed to connect two theories and not the quantities derivable from the theory regardless of the fact that theories belong to the same or two different vacua.

\section{Connecting two different regimes}

As discussed in the introduction, the main non perturbative result of the quadratic gauge was established with the help of additional gauge fixing for one of the gluons. The presence of this additional gauge fixing does reintroduce the Gribov ambiguity for this component but this is the price to be paid for an explicit demonstration of effective masses for the off diagonal gluons. Hence, our aim here is to connect the generating functional corresponding to the effective actions in quadratic gauge with additional Lorenz gauge fixing for one of the gluons to that in the usual Lorenz gauge through the technique of the FFBRST transformation. To do so, we write the effective action of the quadratic gauge with additional gauge fixing for one of the gluons which is as follows

$$
\begin{aligned}
\mathcal{S}_{e f f} & =\mathcal{S}_{Q}+\int d^{4} x\left[\frac{\xi}{2}\left(G^{3}\right)^{2}+G^{3} \partial^{\mu} A_{\mu}^{3}-\overline{d^{3}} \partial^{\mu}\left(D_{\mu} d\right)^{3}\right] \\
& =\mathcal{S}_{Q}+\int d^{4} x\left[\frac{\xi}{2}\left(G^{3}\right)^{2}+G^{3} \partial^{\mu} A_{\mu}^{3}-\overline{d^{3}} \square d^{3}\right]
\end{aligned}
$$

where $\square$ stands for $\partial^{\mu} \partial_{\mu}$ and a set of additional fields $G^{3}, d^{3}, \overline{d^{3}}$ correspond to the additional Lorenz gauge of the diagonal gluon $A^{3}$ and the ghosts $\overline{d^{3}}, d^{3}$ are treated as $S U$ (3) singlets.

As a first approach it is easy to see that the action in Eq. (6) is invariant under the following nilpotent BRST transformation

$$
\begin{aligned}
\delta c^{d} & =\frac{\delta \omega}{2} f^{d b c} c^{b} c^{c} \\
\delta \overline{c^{d}} & =\frac{\delta \omega}{g} F^{d} \\
\delta A_{\mu}^{d} & =\frac{\delta \omega}{g}\left(D_{\mu} c\right)^{d} \\
\delta F^{d} & =0 \\
\delta G^{3} & =0 \\
\delta \square d^{3} & =0 \\
\left(\delta \overline{d^{3}}\right) \square d^{3} & =\frac{\delta \omega}{g} G^{3} \partial^{\mu} D_{\mu} c^{3}
\end{aligned}
$$

where $\delta \omega$ infinitesimal, anticommuting and global parameter. We note that the second last transformation the Eq. (7) can in general be solved for $\delta d^{3}$ explicitly. This set of transformations differs from the usual BRST transformation in the composite form of the last of the Eq. (7), which can always be defined with certainty locally in the same spirit of first five transformations since all fields and their derivatives by construction are well defined at every spacetime point $x$, it is not the differential equation in the $\delta \overline{d^{3}}$ and the $\square$ is an invertible operator (similar concept has appeared in the case of Lagrangian in the literature, see for example Ref. [30]). However, these transformations are not useful for FFBRST technique since the transformations of $G^{3}$ and $\square d^{3}$ are trivial. Therefore, we need to introduce a new set of BRST transformations under which the action (6) is also invariant. This clearly shows that the passage from BRST to FFBRST transformations is non trivial. This conclusion has never been obvious from earlier works. The transformations are as follows

$$
\begin{aligned}
\delta c^{d} & =\frac{\delta \omega}{2} f^{d b c} c^{b} c^{c} \\
\delta \overline{c^{d}} & =\frac{\delta \omega}{g} F^{d} \\
\delta A_{\mu}^{d} & =\frac{\delta \omega}{g}\left(D_{\mu} c\right)^{d} \\
\delta F^{d} & =0 \\
\delta G^{3} & =-\frac{\delta \omega}{g} \overline{d^{3}} \\
\delta \square d^{3} & =\frac{\delta \omega}{g}\left(\partial_{\mu} A^{\mu 3}+\xi G^{3}\right) \\
\left(\delta \overline{d^{3}}\right) \square d^{3} & =\frac{\delta \omega}{g} G^{3} \partial^{\mu} D_{\mu} c^{3}
\end{aligned}
$$

As for the previous transformations, We observe that the second last of Eq. (8) is inhomogeneous wave equation for BRST differential $\delta d^{3}$ with a simple form of the right hand side acting as the source, which surely admits a local solution for $\delta d^{3}(x)$ for given fields. We see that now this set of transformations has become applicable for FFBRST technique as $\delta G^{3}, \delta \square d^{3}$ are non zero. Further, the last three transforma- 
tions in Eq. (8) are not nilpotent, but they satisfy in their compact form the following higher degree closed algebra

$$
\begin{aligned}
\delta^{2}\left(\underline{\left(\delta \overline{d^{3}}\right)} \square d^{3}\right) \square d^{3}=0 \\
\delta^{2}\left(\underline{\left(\delta^{3} \square d^{3}\right)} \square d^{3}\right) \square d^{3}=0 .
\end{aligned}
$$

The remaining one can be easily derived from one of these algebras. Thus, we are prompted to restore the nilpotency and simplify the algebra, to the extent possible. To do so, we express the effective action (6) in terms of a new auxiliary field $B^{3}$,

$$
\begin{aligned}
\mathcal{S}_{e f f}= & \mathcal{S}_{Q}+\int d^{4} x\left[\frac{\xi}{2}\left(G^{3}\right)^{2}+G^{3} \partial^{\mu} A_{\mu}^{3}-\overline{d^{3}} \square d^{3}\right] \\
= & \mathcal{S}_{Q}+\int d^{4} x\left[\frac{-1}{2 \xi}\left(B^{3}\right)^{2}+B^{3} G^{3}\right. \\
& \left.+G^{3} \partial^{\mu} A_{\mu}^{3}-\overline{d^{3}} \square d^{3}\right]
\end{aligned}
$$

The action (10) is invariant under following transformations

$$
\begin{aligned}
\delta c^{d} & =\frac{\delta \omega}{2} f^{d b c} c^{b} c^{c} \\
\delta \overline{c^{d}} & =\frac{\delta \omega}{g} F^{d} \\
\delta A_{\mu}^{d} & =\frac{\delta \omega}{g}\left(D_{\mu} c\right)^{d} \\
\delta F^{d} & =0 \\
\delta G^{3} & =-\frac{\delta \omega}{g} \overline{d^{3}} \\
\delta B^{3} & =-\frac{\delta \omega}{g} \partial^{\mu} D_{\mu} c^{3} \\
\delta \square d^{3} & =\frac{\delta \omega}{g}\left(\partial_{\mu} A^{\mu 3}+B^{3}\right) \\
\left(\delta \overline{d^{3}}\right) \square d^{3} & =\frac{\delta \omega}{g \xi} B^{3} \partial^{\mu} D_{\mu} c^{3}
\end{aligned}
$$

The last three transformation rules satisfy the following algebra

$$
\begin{aligned}
\delta^{2} B^{3} & =0 \\
\delta^{2} \square d^{3} & =0 \\
\delta\left(\underline{\left(\delta \overline{d^{3}}\right) \square d^{3}}\right) & =0
\end{aligned}
$$

We see that first two rules of Eq. (12) are nilpotent and the last one is 'almost' nilpotent now. It is interesting to compare Eqs. (9) and (12). The second of the Eq. (12) implies the following

$\delta^{2} d^{3}=\delta^{n} d^{3}$ for all $n \geq 2$.

This is the unusual example of the BRST transformation with idempotent algebra. Thus, we see that the introduction of $B^{3}$ has made a substantial difference in the algebra of the transformations, with the novel feature of the algebra of the transformation having been made nilpotent through the introduction of an auxiliary field. We shall next achieve the stated connection using these unusual transformations (11) in the FFBRST technique.

Now we briefly outline the procedure for the passage from the BRST transformations to the FFBRST transformations. We start with making the infinitesimal global parameter $\delta \omega$ field dependent by introducing a numerical parameter $\kappa(0 \leq \kappa \leq 1)$ and making all the fields $\kappa$ dependent such that $\phi(x, \kappa=0)=\phi(x)$ and $\phi(x, \kappa=1)=\phi^{\prime}(x)$, the transformed field. The symbol $\phi$ generically describes all the fields $A, c, \bar{c}, F, d^{3}, \bar{d}^{3}, B^{3}, G^{3}$. The BRST transformation in Eq. (11) is then written as

$d \phi=\delta_{b}[\phi(x, \kappa)] \Theta^{\prime}(\phi(x, \kappa)) d \kappa$

where $\Theta^{\prime}$ is a finite field dependent anti-commuting parameter and $\delta_{b}[\phi(x, \kappa)]$ is the form of the transformation for the corresponding field as in Eq. (11). The FFBRST is then constructed by integrating Eq. (13) from $\kappa=0$ to $\kappa=1$ as [6]

$\phi^{\prime} \equiv \phi(x, \kappa=1)=\phi(x, \kappa=0)+\delta_{b}[\phi(0)] \Theta[\phi(x)]$

where $\Theta[\phi(x)]=\int_{0}^{1} d \kappa^{\prime} \Theta^{\prime}[\phi(x, \kappa)]$. Like usual BRST transformation, FFBRST transformation leaves the effective action in Eq. (10) invariant. However, since the transformation parameter is field dependent in nature, FFBRST transformation does not leave the path integral measure, $\mathcal{D} \phi$ invariant and produces a non-trivial Jacobian factor $J$. This $J$ can further be cast as a local functional of fields, $e^{i S_{J}}$ (where the $S_{J}$ is the action representing the Jacobian factor $J$ ) if the following condition is met [6]

$\int \mathcal{D} \phi(x, \kappa)\left[\frac{1}{J} \frac{d J}{d \kappa}-i \frac{d S_{J}}{d \kappa}\right] e^{i\left(S_{J}+\mathcal{S}_{e f f}\right)}=0$.

Thus the procedure for FFBRST may be summarised as (i) calculate the infinitesimal change in Jacobian, $\frac{1}{J} \frac{d J}{d \kappa} d \kappa$ using

$\frac{J(\kappa)}{J(\kappa+d \kappa)}=1-\frac{1}{J(\kappa)} \frac{d J(\kappa)}{d \kappa} d \kappa=\sum_{\phi} \pm \frac{\delta \phi(x, \kappa+d \kappa)}{\delta \phi(x, \kappa)}$

for infinitesimal BRST transformation, + or - sign is for Bosonic or Fermion nature of the field $\phi$ respectively (ii) make an ansatz for $S_{J}$, (iii) then prove the Eq. (15) for this ansatz and finally (iv) replace $J(\kappa)$ by $e^{i S_{J}}$ in the generating functional

$$
W=\int \mathcal{D} \phi(x) e^{i S_{e f f}(\phi)}=\int \mathcal{D} \phi(x, \kappa) J(\kappa) e^{i S_{e f f}(\phi(x, \kappa))} .
$$


Setting $\kappa=1$, this would then provide the new effective action $S_{\text {eff }}^{\prime}=S_{J}+S_{\text {eff }}$.

Now we proceed to construct a FFBRST transformation with an appropriate parameter to connect the generating functionals in the quadratic gauge with additional Lorenz gauge for the diagonal gluon $A^{3}$ and the Lorenz gauge. We construct the finite field dependent parameter as

$$
\begin{aligned}
& \Theta^{\prime}[\phi(k)] \\
& \quad=-i \int d^{4} x\left[\overline{c^{a}}\left(\gamma_{2} A_{\mu}^{a} A^{\mu a}+\gamma_{3} \partial_{\mu} A^{\mu a}\right)+\gamma_{1} G^{3} \square d^{3}\right]
\end{aligned}
$$

The $\gamma_{1}, \gamma_{2}$ and $\gamma_{3}$ are constant parameters and $\Theta^{\prime 2}=0$. Group index $a$ is summed over. This FFBRST transformation is particularly different among others [15-28] due to the unique form of transformations (11) and by the fact that the field dependent parameter in Eq. (18) contains two ghosts $\bar{c}, d$ with two different transformation properties unlike others where there is only one ghost. We now calculate the change in the Jacobian $\frac{1}{J} \frac{d J}{d \kappa}$ due to the FFBRST with the parameter in Eq. (18), under which the measure changes $\mathcal{D} \phi(\kappa) \rightarrow J(\kappa) \mathcal{D} \phi(\kappa)$ as

$$
\begin{aligned}
\frac{1}{J} \frac{d J}{d k}= & -\frac{1}{g} \int d^{4} x\left(\frac{\delta \Theta^{\prime}}{\delta A_{\mu}^{a}}\left(D_{\mu} c\right)^{a}+\frac{\delta \Theta^{\prime}}{\delta \partial^{\mu} A_{\mu}^{a}} \partial^{\mu}\left(D_{\mu} c\right)^{a}\right. \\
& -\frac{\delta\left(\Theta^{\prime} f^{a b c} c^{b} c^{c}\right)}{2 \delta c^{a}}-\frac{\delta \Theta^{\prime}}{\delta \overline{c^{a}}} F^{a} \\
& \left.-\frac{\delta \Theta^{\prime}}{\delta \square d^{3}}\left(\partial A^{\mu 3}+B^{3}\right)-\frac{\delta \Theta^{\prime}}{\delta G^{3}} \overline{d^{3}}\right) \\
= & \frac{i}{g} \int d^{4} x\left(2 \gamma_{2} \overline{c^{a}}\left(D_{\mu} c\right)^{a} A^{\mu a}+\gamma_{3} \overline{c^{a}} \partial^{\mu}\left(D_{\mu} c\right)^{a}\right. \\
& -F^{a}\left(\gamma_{2} A_{\mu}^{a} A^{\mu a}+\gamma_{3} \partial_{\mu} A^{\mu a}\right) \\
& \left.-\gamma_{1} G^{3}\left(\partial_{\mu} A^{\mu 3}+B^{3}\right)+\gamma_{1} \overline{d^{3}} \square d^{3}\right)
\end{aligned}
$$

Since $\frac{1}{J} \frac{d J}{d k}$ does not contain terms with $\Theta^{\prime}$ as multiplicative factor, the $\kappa$ dependence in $S_{J}(\kappa)$ is multiplicative [6]. This implies that the fields in the ansatz for the $S_{J}$ can be taken to be $\kappa$ independent. With this fact in mind, we make the following ansatz for the $S_{J}$ to compensate the Jacobian contribution of FFBRST transformation

$$
\begin{aligned}
S_{J}[\phi, \kappa]= & \frac{1}{g} \int d^{4} x\left(\alpha_{1}(\kappa) F^{a} A_{\mu}^{a} A^{\mu a}+2 \alpha_{2}(\kappa) \overline{c^{a}} A^{\mu a}\left(D_{\mu} c\right)^{a}\right. \\
& +\alpha_{3}(\kappa) F^{a} \partial_{\mu} A^{\mu a}+\alpha_{4}(\kappa) \overline{c^{a}} \partial^{\mu}\left(D_{\mu} c\right)^{a} \\
& \left.+\alpha_{5}(\kappa) G^{3}\left(\partial^{\mu} A_{\mu}^{3}+B^{3}\right)+\alpha_{6}(\kappa) \overline{d^{3}} \square d^{3}\right)
\end{aligned}
$$

where $\alpha_{j}(\kappa), j=1, \ldots, 6$, are arbitrary functions with initial condition $\alpha_{i}(\kappa=0)=0$ while the fields themselves are $\kappa$ independent. We calculate,

$$
\begin{aligned}
& i \frac{d S_{J}}{d k}=\frac{i}{g} \int d^{4} x\left[\dot{\alpha}_{1} F^{a} A_{\mu}^{a} A^{\mu a}+2 \dot{\alpha_{2}} \overline{c^{a}} A^{\mu a}\left(D_{\mu} c\right)^{a}\right. \\
& +\dot{\alpha_{3}} F^{a} \partial_{\mu} A^{\mu a}+\dot{\alpha_{4}} \overline{c^{a}} \partial^{\mu}\left(D_{\mu} c\right)^{a} \\
& \left.+\dot{\alpha_{5} G^{3}}\left(\partial^{\mu} A_{\mu}^{3}+B^{3}\right)+\dot{\alpha_{6}} \overline{d^{3}} \square d^{3}\right]
\end{aligned}
$$

In order to satisfy the condition in Eq. (15), the following equation must be obeyed

$$
\begin{aligned}
& \int \mathcal{D} \phi[x, \kappa]\left[F^{a} A_{\mu}^{a} A^{\mu a}\left(-\gamma_{2}-\dot{\alpha}_{1}\right)+2 \overline{c^{a}} A^{\mu a}\left(D_{\mu} c\right)^{a}\left(\gamma_{2}-\dot{\alpha}_{2}\right)\right. \\
& \quad+F^{a} \partial_{\mu} A^{\mu a}\left(-\gamma_{3}-\dot{\alpha_{3}}\right)+\overline{c^{a}} \partial^{\mu}\left(D_{\mu} c\right)^{a}\left(\gamma_{3}-\dot{\alpha}_{4}\right) \\
& +\left(-\gamma_{1}-\dot{\alpha}_{5}\right) G^{3}\left(\partial^{\mu} A_{\mu}^{3}+B^{3}\right) \\
& \left.+\left(\gamma_{1}-\dot{\alpha}_{6}\right) \overline{d^{3}} \square d^{3}\right] e^{i\left(S_{e f f}+S_{J}\right)}=0
\end{aligned}
$$

which gives the following relation among parameters

$\dot{\alpha}_{1}=-\dot{\alpha}_{2}=-\gamma_{2}$

$\dot{\alpha}_{3}=-\dot{\alpha}_{4}=-\gamma_{3}$

$\dot{\alpha}_{5}=-\dot{\alpha}_{6}=-\gamma_{1}$.

The Eqs. (23) have the obvious solutions

$$
\begin{aligned}
& \alpha_{1}=-\alpha_{2}=-\gamma_{2} \kappa ; \alpha_{3}=-\alpha_{4}=-\gamma_{3} \kappa, \\
& \alpha_{5}=-\alpha_{6}=-\gamma_{1} \kappa
\end{aligned}
$$

We choose the arbitrary parameters $\gamma_{1}=1, \gamma_{2}=1, \gamma_{3}=-1$ in Eq. (24). Thus, the additional Jacobian contribution at $\kappa=1$ is

$$
\begin{aligned}
S_{J}= & \int d^{4} x\left(-F^{a} A_{\mu}^{a} A^{\mu a}+2 \overline{c^{a}} A^{\mu a}\left(D_{\mu} c\right)^{a}\right. \\
& +F^{a} \partial_{\mu} A^{\mu a}-\overline{c^{a}} \partial^{\mu}\left(D_{\mu} c\right)^{a} \\
& \left.-G^{3}\left(\partial^{\mu} A_{\mu}^{3}+B^{3}\right)+\overline{d^{3}} \square d^{3}\right) .
\end{aligned}
$$

Adding this Jacobian contribution, $S_{J}$ to the $\mathcal{S}_{\text {eff }}$ in Eq. (10) we obtain at $\kappa=1$ the Lorenz gauge as follows

$$
\begin{aligned}
\mathcal{S}_{e f f}+S_{J}= & \int d^{4} x\left[-\frac{1}{2 \xi}\left(B^{3}\right)^{2}+\frac{\zeta}{2} F^{a 2}+F^{a} \partial_{\mu} A^{\mu a}\right. \\
& \left.-\overline{c^{a}} \partial^{\mu}\left(D_{\mu} c\right)^{a}\right]=S_{L}
\end{aligned}
$$

Here the term $\frac{1}{\xi}\left(B^{3}\right)^{2}$ is redundant which can be put to zero by using EOM for $B^{3}$. Now, we may further apply second FFBRST such that $\zeta \rightarrow \zeta^{\prime}$ in the same Lorenz gauge by well known methods [6]. We may summarize this symbolically as the conversion from one theory to another,

$$
\begin{aligned}
Z_{\text {eff }} & =\int \mathcal{D} \phi e^{i \mathcal{S}_{e f f}} \stackrel{\text { FFBRST }}{\longrightarrow} \int \mathcal{D} \phi^{\prime}(\kappa) e^{i\left(\mathcal{S}_{\text {eff }}+S_{J}\right)} \\
& =\int \mathcal{D} \phi^{\prime} e^{i S_{L}}=Z_{L},
\end{aligned}
$$

Thus, we have connected two theories with two different regimes of applicability. This is a connection also between theories with and without propagating ghosts. 


\section{Conclusion}

The spirit of BRST invariance was to establish the unitarity of the S-matrix in gauge theories whose gauge fixed versions contain ghost degrees of freedom. This technique was substantially extended in the FFBRST approach to permit field redefinitions transforming the effective action with one possible gauge fixing to that of another. In some of the recent earlier work the interesting features of a purely quadratic gauge condition without the usual Lorenz condition have been studied and shown to lead to several interesting properties of the non-perturbative QCD vacuum in the IR limit. At first site the effective degrees of freedom entering here, the off diagonal gluons with masses, appear unrelated to those entering the perturbation theory calculations and which are compatible with the elegant UV properties of Yang-Mills theories. In this paper we have resorted to the FFBRST technique to establish a direct formal connection between the two varieties of the QCD effective lagrangians. Several technical difficulties are encountered in this process and it has required us to make suitable extensions to the FFBRST method. In particular a new auxiliary field is required to ensure nilpotency of the modified BRST transformations. The resulting field redefinitions which connect the degrees of freedom capturing the IR behaviour of QCD vacuum with those of the UV version suitable to perturbative computations need to be studied further. Also, the extensions of the FFBRST technique proposed here can be put to use for other similar problems.

Acknowledgements We acknowledge the fruitful discussion with Prof. Urjit A. Yajnik. This work is partially supported by Department of Science and Technology, Govt. of India under National Postdoctoral Fellowship scheme with File No. 'PDF/2017/000066'. One of us (BPM) acknowledges the support from Physics Department, IIT-Bombay for a visitation during which the work was initiated.

Open Access This article is distributed under the terms of the Creative Commons Attribution 4.0 International License (http://creativecomm ons.org/licenses/by/4.0/), which permits unrestricted use, distribution, and reproduction in any medium, provided you give appropriate credit to the original author(s) and the source, provide a link to the Creative Commons license, and indicate if changes were made.

Funded by SCOAP ${ }^{3}$.

\section{References}

1. K. Shizuya, Nucl. Phys. B 109, 397-420 (1976)

2. S. Weinberg, Phys. Lett. B 91, 51-55 (1980)

3. A. Das, Pramana 16, 409-416 (1981)

4. A. Das, M.A. Namazie, Phys. Lett. B 99, 463-466 (1981)

5. S.D. Joglekar, Phys. Rev. D 10, 4095 (1974)

6. S.D. Joglekar, B.P. Mandal, Phys. Rev. D 51, 1919 (1995)

7. H. Raval, U.A. Yajnik, Phys. Rev. D 91(8), 085028 (2015)

8. H. Raval, U.A. Yajnik, Springer Proc. Phys. 174, 55 (2016)

9. Haresh Raval, Eur. Phys. J. C 76, 243 (2016)

10. G.t Hooft, Nucl. Phys. B 190, 455 (1981)

11. H.S. Chan, M.B. Halpern, Phys. Rev. D 33, 540 (1986)

12. S. Weinberg, in The quantum theory of fields. Vol. 2: Modern applications (Cambridge University Press, Cambridge, 1996)

13. C. Becchi, A. Rouet, R. Stora, Commun. Math. Phys. 42, 127 (1975)

14. C. Becchi, A. Rouet, R. Stora, Ann. Phys. 98, 287 (1976)

15. S.D. Joglekar, B.P. Mandal, Int. J. Mod. Phys. A 17, 1279 (2002)

16. R. Banerjee, B.P. Mandal, Phys. Lett. B 488, 27 (2000)

17. S. Upadhyay, S.K. Rai, B.P. Mandal, J. Math. Phys. 52, 022301 (2011)

18. S. Deguchi, V.K. Pandey, B.P. Mandal, Phys. Lett. B 756, 394 (2016)

19. S. Upadhyay, B.P. Mandal, Eur. Phys. J. C 72, 2065 (2012)

20. S. Upadhyay, B.P. Mandal, Phys. Lett. B 744, 231 (2015)

21. B.P. Mandal, S.K. Rai, S. Upadhyay, Eur. Phys. Lett. 92, 21001 (2010)

22. M. Faizal, B.P. Mandal, S. Upadhyay, Phys. Lett. B 721, 159 (2013)

23. M. Faizal, B.P. Mandal, S. Upadhyay, Phys. Lett. B 738, 201 (2014)

24. S. Upadhyay, Phys. Lett. B 727, 293 (2013)

25. S. Upadhyay, Eur. Phys. Lett. 105, 21001 (2014)

26. S. Upadhyay, Eur. Phys. Lett. 104, 61001 (2013)

27. P.Y. Moshin, A.A. Reshetnyak, Phys. Lett. B 739, 110 (2014)

28. P.Y. Moshin, A.A. Reshetnyak, Nucl. Phys. B 888, 92 (2014)

29. P. Hoyer, Nucl Phys. B Proc. Suppl. 117, 367-369 (2003)

30. D. Zwanziger et al., Phys. Rep. 520(4), 175-251 (2012) 